\title{
UNITARY CONGRUENCES AND POSITIVE BLOCK-MATRICES
}

\section{ANTOINE MHANNA}

Abstract. In this note we give some two by two block matrices $M=\left(\begin{array}{cc}A & X \\ X^{*} & B\end{array}\right)$ where $M$ and $M^{\prime}=\left(\begin{array}{cc}A & X^{*} \\ X & B\end{array}\right)$ are unitarily congruent. We also generalize a class of positive semi-definite block-matrices satisfying the inequality $\|M\| \leqslant\|A+B\|$ for all symmetric norms.

Mathematics subject classification (2010): 15A21, 15A60, 15A42.

Keywords and phrases: Symmetric norm, positive block-matrices, positive partial transpose.

\section{REFERENCES}

[1] F. Zhang, Matrix Theory Basic Results and Techniques, $2^{\text {nd }}$ Edition, (Universitext) Springer, 2011.

[2] J. C. BOURIN, E. Y. LEE, Unitary orbits of Hermitian operators with convex or concave functions, Bull. London Math. Soc. 44, 6 (2012), 1085-1102.

[3] J. C. Bourin, A. Mhanna, Positive block matrices and numerical ranges, C. R. Math. Acad. Sci. Paris 355, 10 (2017), 1077-1081.

[4] A. I. SingH, Role of partial transpose and generalized Choi maps in quantum dynamical semigroups involving separable and entangled states, Electronic Journal of Linear Algebra 29 (2015), 156-193.

[5] M. Gumus, J. LiU, S. RaOUAFI, T. Y. TAM, Positive semi-definite $2 \times 2$ block matrices and norm inequalities, Linear Algebra Appl. 551 (2018), 83-91.

[6] A. Mhanna, On symmetric norm inequalities and positive definite block-matrices, Math. Inequal. Appl. 21, 1 (2018), 133-138.

[7] M. Lin, H. Wolkowicz, Hiroshima's theorem and matrix norm inequalities, Acta Sci. Math. (Szeged) 81, 1-2 (2015), 45-53. 